\title{
Pronóstico estacional de precipitaciones mejorado usando una estimación óptima de la NAO invernal
}

https://doi.org/10.31978/639-19-010-0.649

\author{
Eroteida Sánchez García ${ }^{1}$ (esanchezg@aemet.es) \\ José Voces Aboy ${ }^{1}$ (jvocesa@aemet.es) \\ Beatriz Navascués Fernández-Victorio² (bnavascuesf@aemet.es) \\ Ernesto Rodríguez Camino³ (erodriguezc@aemet.es) \\ ${ }^{1}$ AEMET / Delegación Territorial en Cantabria \\ ${ }^{2}$ AEMET / Dirección de Estrategia, Planificación y Desarrollo Comercial \\ ${ }^{3}$ AEMET / Dirección de Producción e Infraestructuras / Área de Evaluación y Modelización del Clima
}

\section{RESUMEN}

Actualmente, la mayoría de los sistemas de predicción estacional basados en modelos AOCGC (Atmospheric Ocean Coupled Global Circulation) no tienen la suficiente habilidad para predecir el índice NAO (Oscilación del Atlántico Norte), y, por tanto, tampoco tienen habilidad para predecir la precipitación del periodo invernal sobre el sur de Europa. Por otra parte, CoHEN y Jones (2011) han demostrado que parte de la variabilidad de la NAO en los meses del invierno puede ser forzada externamente por el avance de la cobertura nivosa a finales del otoño.

En este estudio se intenta obtener una estimación óptima de este modo dominante de variabilidad extratropical y su incertidumbre, utilizando estimaciones a priori de NAO pronosticadas a partir de sistemas de predicción estacionales operacionales y empíricos.

La suposición de la equiprobabilidad de los miembros del conjunto generalmente se aplica a conjuntos atmosféricos generados a partir de un único modelo. La ponderación de los miembros de un sistema de pronóstico estacional no es una tarea trivial y con frecuencia depende de la aplicación particular o región objetivo del pronóstico. Teniendo en cuenta que nuestro objetivo final es mejorar la precipitación invernal en la península ibérica, la función densidad de probabilidad de la precipitación pronosticada se obtiene ponderando los miembros del ensemble del sistema dinámico siguiendo la idea original de Dobrynin et al. (2016) y utilizando una función de densidad de probabilidad de la NAO prevista por un sistema empírico. Mostramos la notable mejora de los pronósticos de precipitación invernal sobre nuestra región de interés cuando los miembros son ponderados en comparación con el enfoque habitual basado en la equiprobabilidad de los miembros del conjunto. Las relaciones empíricas alternativas también se exploran. En resumen, con este punto de vista, estamos tratando de explorar una forma de mejorar el pronóstico estacional para alguna aplicación específica en un área específica usando la información que tenemos disponible.

PALABRAS CLAVE: predicción; estacional; precipitación; península ibérica; estimación índice NAO invernal. 


\section{INTRODUCCIÓN}

Aunque los sistemas operacionales de predicción estacional (SFS, Seasonal Forecasting System) han demostrado poca o ninguna habilidad en las latitudes medias europeas, el trabajo reciente ha demostrado que algunos SFS basados en modelos dinámicos muestran una habilidad notable en la predicción de NAO de invierno (por ejemplo, SCAIFE et al., 2014), índice que se sabe que tiene influencia en el clima de la fachada del suroeste de Europa.

\section{EOF: North Atlantic Oscillation (NAO)}

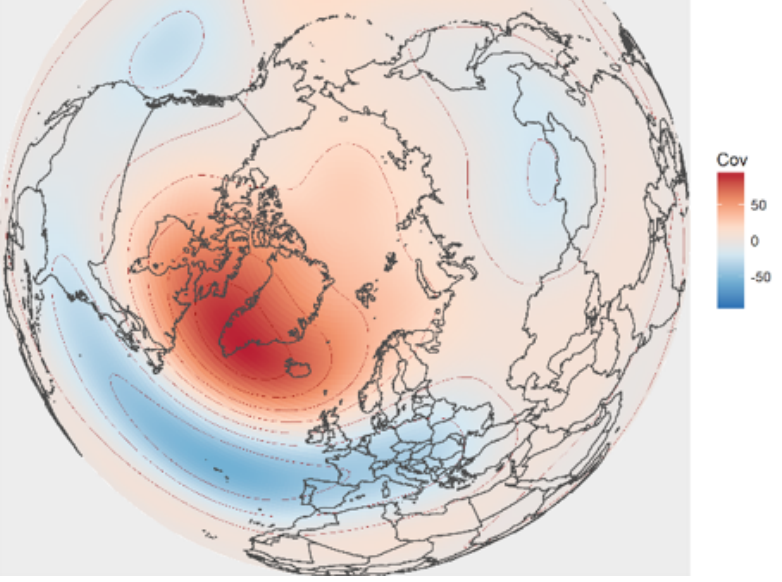

Figura 1. Patrón de teleconexión NAO.
Por otro lado, COHEN y Jones (2011) han demostrado que parte de la variabilidad invernal de la NAO puede verse forzada externamente por el avance de la capa de nieve boreal en otoño. Dobrynin et al. (2018) también han demostrado que la habilidad de un SFS se puede mejorar significativamente refinando un conjunto dinámico mediante submuestreo basado en la predicción empírica de la NAO.

Este trabajo utiliza una estimación óptima de la NAO invernal (combinando información de la NAO prevista por un modelo dinámico y de un modelo empírico) como métrica para modificar la ponderación de los diferentes miembros del ensemble del SFS. Comparamos la habilidad de un conjunto de SFS usando miembros del conjunto ponderados y no ponderados.

\section{2. ¿CÓMO PREDICEN LOS SISTEMAS DE PREDICCIÓN ESTACIONALES LA NAO INVERNAL?}

Se ha analizado la capacidad de simular la NAO de invierno de cuatro sistemas operacionales de predicción estacional almacenados en Copernicus Climate Data Store:

- ECMWF System 5

- ECMWF System 4

- Met Office System 12

- Météo-France System 5

El reanálisis ERA-Interim (ERAI) ha sido utilizado para obtener patrones EOF (Empirical Orthogonal Function) y series temporales del

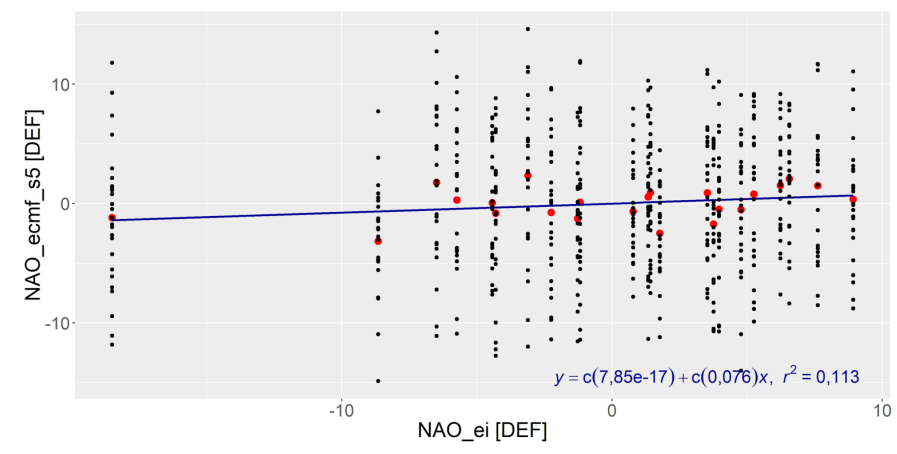

Figura 2: Correlación NAO invernal. índice NAO de forma similar a BUTLER et al. (2016). Para cada sistema de predicción, se obtiene el índice NAO para cada miembro del ensemble del SFS proyectando la anomalía del geopotencial en $500 \mathrm{hPa}$ sobre el primer EOF. Para evaluar los resultados, se compara la serie temporal del índice NAO previsto por cada sistema con el del ERAI.

En la figura 2 podemos ver la correlación entre la NAO invernal obtenida a partir de datos del ERA Interim y la obtenida con los datos del modelo ECMF-S5. 
El índice NAO invernal previsto por el sistema empírico a escala estacional, SClimWaRe, desarrollado en AEMET y basado en la teleconexión de la cubierta de nieve en otoño (VocEs et al., 2016) también ha sido verificado contra el índice NAO del ERAI. El periodo de verificación está restringido a 1997-2015 debido a la disponibilidad de los productos de cobertura de nieve satelital de entrada en S-ClimWaRe. En la figura 3 mostramos una tabla con los resultados para todos los SFS considerados.

\begin{tabular}{|l|c|c|}
\hline \multicolumn{1}{|c|}{$\begin{array}{c}\text { SISTEMA } \\
\text { DE PREDICCIÓN } \\
\text { ESTACIONAL }\end{array}$} & $\begin{array}{c}\text { R } \\
\text { (NAO media prevista por } \\
\text { el sistema - NAO ERAI) (1997-2015) }\end{array}$ & $\begin{array}{c}\text { spread/RMSE } \\
\text { (1997-2015 excluyendo el } \\
\text { valor extremo de NAO 2009) }\end{array}$ \\
\hline ECMWF System 5 & 0,37 & 1,16 \\
\hline ECMWF System 4 & 0,14 & 1,09 \\
\hline Met Office System 12 & 0,09 & 1,01 \\
\hline Météo-France System 5 & 0,12 & 1,04 \\
\hline Modelo empírico S-ClimWaRe & 0,31 & 1,03 \\
\hline
\end{tabular}

Figura 3. Resultados de la verificación del índice NAO.

ElECMWF-S5 y el sistema empírico S-ClimWaRe son los sistemas de predicción estacional que presentan la correlación más alta con la NAO de invierno de ERAI. Sin embargo, el sistema ECMWF-S5 parece ser ligeramente sobredispersivo.

\section{ESTIMACIÓN ÓPTIMA DE LA NAO MEDIA DE DEF}

Aplicamos la teoría de estimaciones estadísticas (KALNAY, 2003) para obtener una estimación óptima del índice NAO invernal [DEF] y de su incertidumbre.

Considerando como first guess los valores de la NAO previstos por un modelo dinámico operacional, este se puede corregir asimilando otras estimaciones del índice NAO, por ejemplo aquellas obtenidas mediante relaciones empíricas o teleconexiones.

Se asumen como gaussianos los errores de todas las estimaciones a priori para calcular la pdf de la estimación óptima de la NAO. El método es similar al enfoque bayesiano utilizado por CoelHo y Pezulli (2004) para estimar la distribución del pronóstico estacional del índice ENSO.

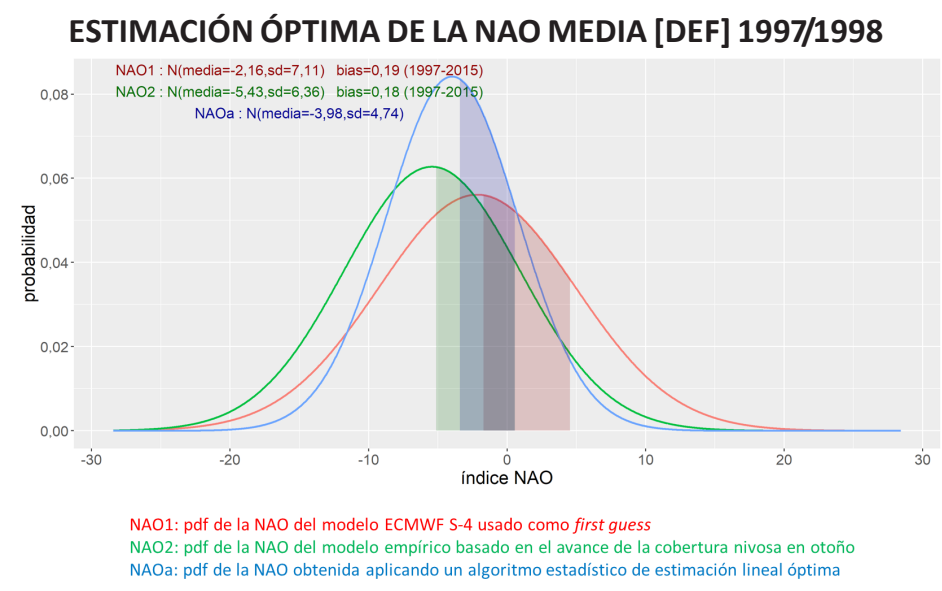

Figura 4. Estimación óptima de la NAO usando ECMWF-S4.
En la figura 4 vemos las funciones gaussianas de la NAO obtenidas en el caso de combinar el modelo dinámico ECMWF-S4 con el modelo empírico S-ClimWaRe.

La función de probabilidad del índice NAO obtenida aplicando el algoritmo de estimación lineal óptima (color azul en la figura 4) sirve de base para ponderar los diferentes miembros del ensemble del modelo dinámico en el postproceso de las predicciones de precipitación acumulada en el periodo invernal que nos interesa. 


\section{VERIFICACIÓN DE LA PRECIPITACIÓN PREVISTA POR LOS MODELOS PESANDO LOS MIEMBROS CON LA ESTIMACIÓN ÓPTIMA DE LA NAO}

La pdf del first guess para la precipitación acumulada en los meses de NDEFM (igual peso para cada miembro del ensemble) se modifica asignando un peso diferente a cada miembro, en función de la métrica basada en la mejor estimación del índice NAO.

El procedimiento produce una mejora notable de los pronósticos de precipitación durante el invierno extendido (NDJFM) para el periodo de hindcast (1997-2015) en todos los sistemas de pronóstico estacional de Copernicus presentados anteriormente.

Las figuras presentadas a continuación corresponden a los pronósticos estacionales de precipitación producidos cuando se toma como first guess la salida del modelo ECMWF-S5. En la verificación, como base de observaciones se toma el conjunto de datos E-Obs. Se usa la media del conjunto para el cálculo de los índices deterministas y para los probabilísticos los terciles inferior y superior.

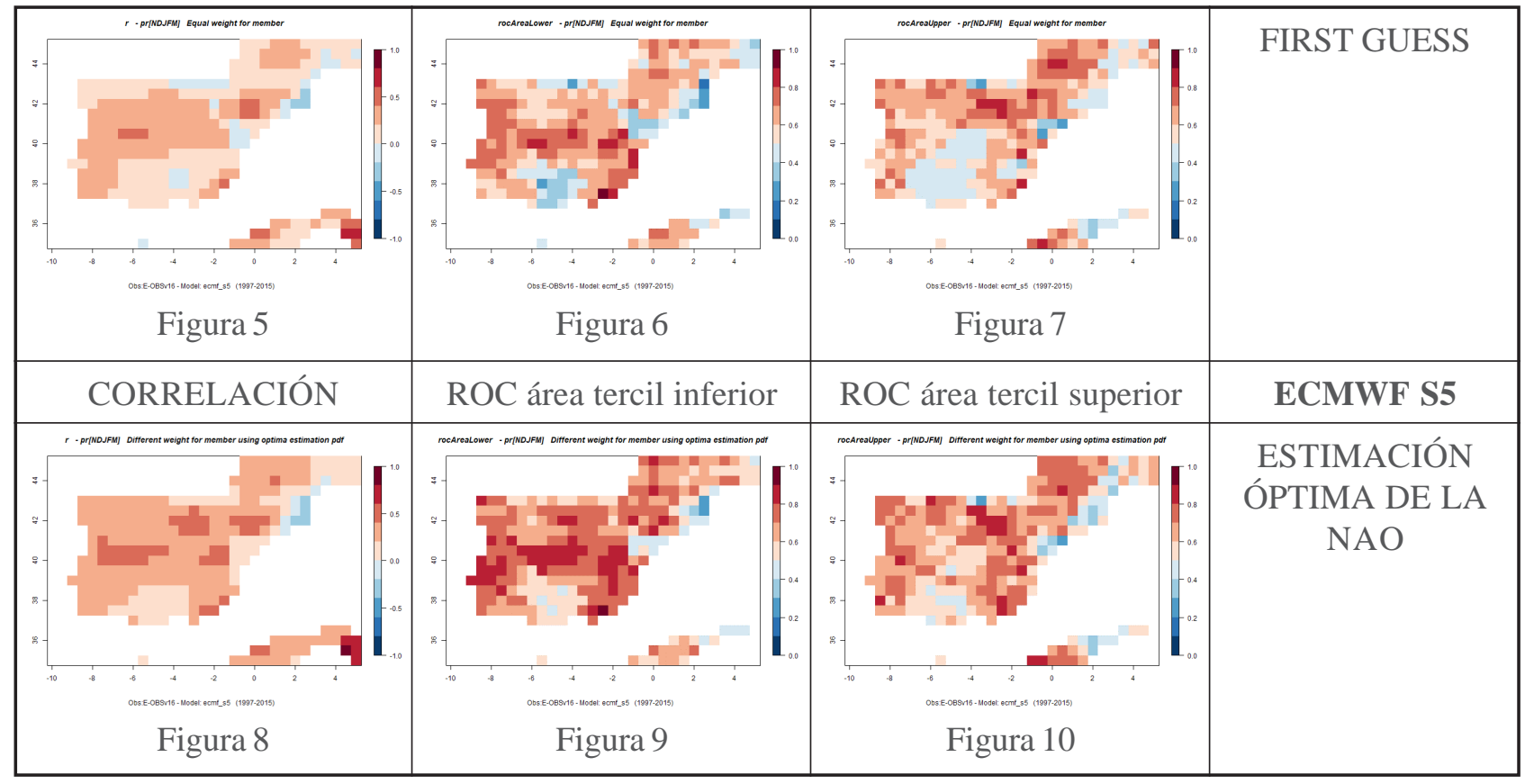

Los resultados confirman que una predicción correcta de la NAO invernal es clave para obtener pronósticos de precipitación hábiles en esta región.

\subsection{Impacto de la NAO del first guess: ECMWF S4 vs ECMWF S5}

La habilidad del first guess para predecir la NAO tiene un impacto positivo no solo en los pronósticos de precipitación del propio sistema, sino también en la mejora adicional producida por este método de estimación óptima de la NAO (como se ve que ha sucedido con la reciente actualización del sistema de predicción estacional del ECMWF).

La figura 12 muestra la correlación de la precipitación invernal pronosticada por ECMWF-S4 pesando los miembros del ensemble con la función de densidad de la NAO obtenida con el método de estimación óptima (combinando el propio modelo ECMWF-S4 con el modelo empírico S-ClimWaRe). Si comparamos con la figura 8, vemos la mejoría obtenida si usamos la última versión del modelo (ECMWF-S5). Lo mismo sucede con el resto de índices de verificación calculados. 


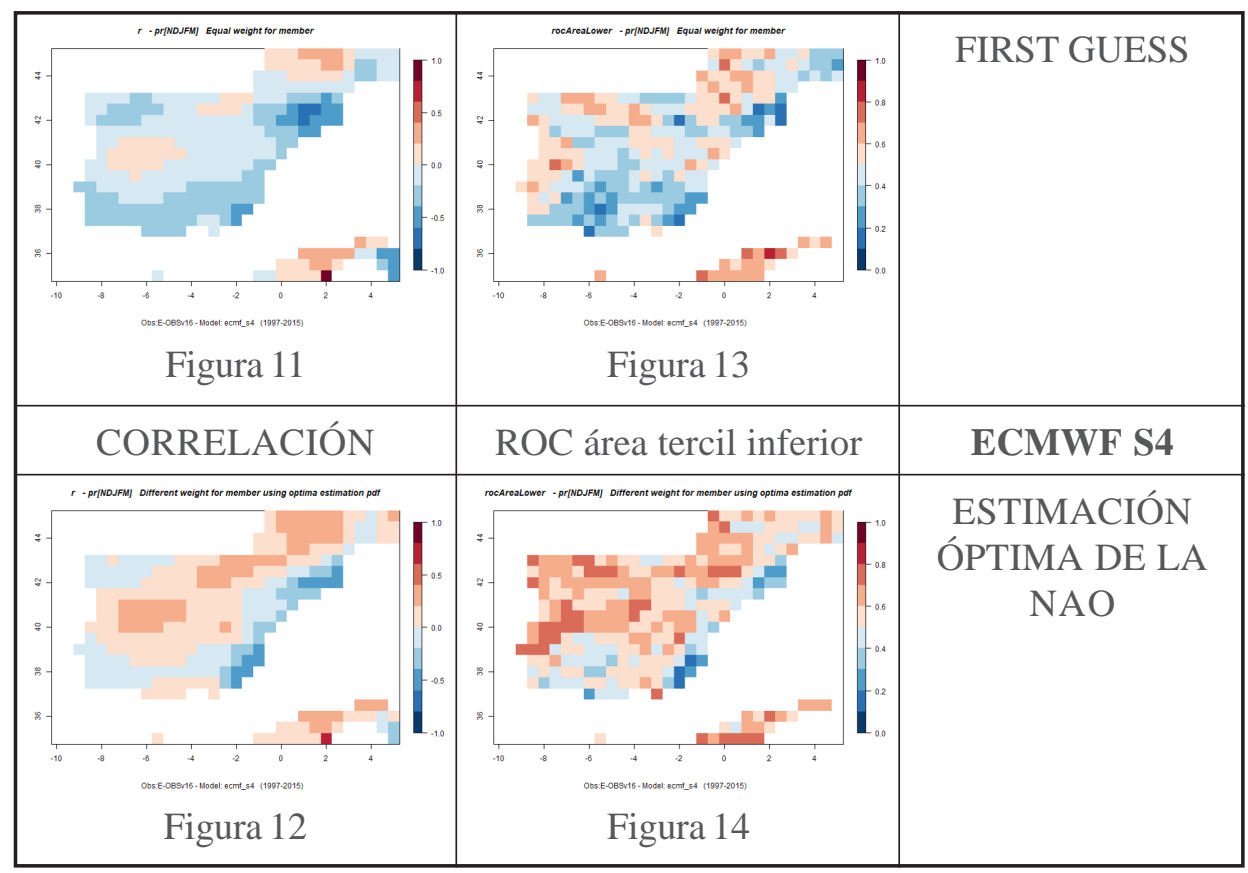

\section{CONCLUSIONES}

En base a los resultados obtenidos, podemos decir que la ponderación de los miembros de los sistemas ECMWF S5 y S4 utilizando una métrica basada en la NAO prevista por cada miembro y la pdf de la estimación óptima (combinando información del sistema del ECMWF y de relaciones empíricas) mejora la habilidad de los sistemas para predecir la precipitación acumulada en el periodo NDJFM sobre la península ibérica.

Además, la mejora del sistema ECMWF S5 (frente al S4) se traduce inmediatamente en la mejora del sistema ECMWF ponderando los miembros. Otros sistemas operacionales probados (Météo-France y Met Office) exhiben la misma mejora cuando se ponderan sus miembros.

Respecto al trabajo futuro, se pueden abrir varias líneas de investigación.

- La combinación de varios sistemas dinámicos para una mejor estimación de NAO.

- Mejoras en el modelo empírico.

- El uso de periodos de verificación más largos.

\section{REFERENCIAS}

ButLer, A. H. et al., 2016. The Climate-system Historical Forecast Project: do stratosphere-resolving models make better seasonal climate predictions in boreal winter? Quart. J. of the R. Met. Soc., vol. 142, 1413-1427.

Coelho, C. A. S. y Pezzulli, S., 2004. Forecast Calibration and Combination: A Simple Bayesian Approach for ENSO. J. of Climate, vol. 17, 1504-1516.

Cohen, J. y Jones, J., 2011. A new index for more accurate winter predictions. Geophys. Res. Lett., 38, L21701, doi:10.1029/2011GL049626. 
Dobrynin, M. et al., 2018. Improved Teleconnection-Based Dynamical Seasonal Predictions of Boreal Winter. Geophys. Res. Lett., vol.45, 3605-3614.

Kalnay, E., 2003. Atmospheric modelling, Data Assimilation and Predictability. Cambridge University Press.

SCAIFE, A. A. et al., 2014. Skillful longrange prediction of European and North American winters. Geophys. Res. Lett., vol. 41, 2514-2519.

Voces, J. et al., 2016. Sistema estadístico de predicción estacional para la gestión de los embalses en España. Nota Técnica no 21 de AEMET. http://www.aemet.es/es/conocermas/recursos_en_linea/ publicaciones_y_estudios/publicaciones/detalles/NT_21_AEMET. 